\title{
Suture Line Dehiscence after Endovascular Aneurysm Repair in a Patient with Marfan Syndrome
}

\author{
Hirofumi Kasahara, MD, PhD, ${ }^{1}$ Norimasa Haijima, $M D,{ }^{2}$ and Takashi Hachiya, $M D, \mathrm{PhD}^{1}$
}

\begin{abstract}
A 68-year-old man with Marfan syndrome developed de-novo leakage after endovascular aneurysm repair of a Dacron graft. Findings at subsequent reoperation suggested that the rebound force on the endograft generated by marked longitudinal deformation in the Dacron graft may have placed stress on the suture line, leading to partial dehiscence. The Dacron graft seemed to provide a stable proximal landing zone. However, the strong tendency of some endograft devices to return to their original shape may apply stress that affected weakened regions of the native aorta.
\end{abstract}

Keywords: abdominal aortic aneurysm, endovascular aneurysm repair, Marfan syndrome

\section{Introduction}

Endovascular aneurysm repair (EVAR) is still a controversial procedure in patients with Marfan syndrome or other connective tissue diseases. Here we report a rare case of suture line dehiscence of a prior Dacron graft 1 year after EVAR. The reason for this result could be the generation of a rebound force by deploying a stent graft into a severely kinked prosthesis.

\section{Case Report}

The patient was a 68-year-old man with Marfan syndrome. At 50 years of age, he was treated for the rupture of an infrarenal abdominal aortic aneurysm by replacement using a knitted Dacron graft. His past history included a total of three laparotomies and the modified

\footnotetext{
${ }^{1}$ Department of Cardiovascular Surgery, Kawasaki Municipal Hospital, Kawasaki, Kanagawa, Japan

${ }^{2}$ Department of Cardiovascular Surgery, National Hospital Organization Saitama Hospital, Wako, Saitama, Japan
}

Received: February 6, 2017; Accepted: April 3, 2017

Corresponding author: Hirofumi Kasahara, MD, PhD. Department of Cardiovascular Surgery, Kawasaki Municipal Hospital, 12-1 Shinkawadori, Kawasaki-ku, Kawasaki, Kanagawa 2100013, Japan

Tel: +81-44-233-5521, Fax: +81-44-245-9600

E-mail: kasa7777@gmail.com
Bentall procedure for aortic root dilatation.

At 65 years of age, an abdominal pseudoaneurysm caused by leakage from the distal anastomosis of the Dacron graft was detected by computed tomography (CT), and he was referred to Saitama Hospital. Follow-up CT revealed enlargement of the pseudoaneurysm sac. Therefore, an endovascular repair was scheduled to cover the site of leak without the need of a fourth laparotomy. Preoperative CT revealed that the mid-portion of the Dacron graft was kinked at a 120-degree angle (Fig. 1). Therefore, we deployed an Excluder endograft $(26 \mathrm{~mm} \times 180 \mathrm{~mm}$; W. L. Gore \& Associates, Flagstaff, AZ, USA). The proximal end of the Excluder was placed beyond the kink in the Dacron graft with the distal end reaching the normal external iliac artery. Because a type 1a endoleak was suspected just after deployment of the endograft, an additional stent graft $(26 \mathrm{~mm} \times 33 \mathrm{~mm})$ was deployed proximally. The proximal tip of this stent graft was located close to the proximal anastomosis of the Dacron graft. Postoperative CT confirmed the absence of the endoleak.

One year after implantation of the endograft, de-novo leakage from the proximal anastomosis of the Dacron graft was detected along with further enlargement of the aneurysm sac (Fig. 2). Therefore, we decided to perform an open surgery via a midline abdominal incision. Interrenal cross-clamping (the aortic cross-clamp was placed below one renal artery and above the other renal artery) of the proximal aorta was used to maintain hemilateral renal perfusion. ${ }^{1)}$ Suture line dehiscence was detected at the posterior part of the proximal anastomosis of the Dacron graft, corresponding to the site identified by CT. This dehiscence was the cause of the blood leak into the space created by closure of the aneurysm around the Dacron graft in the first surgery. After the proximal anastomosis was completely released from the native aorta, the kink in the Dacron prosthesis was immediately reduced by the rebound force from the implanted endograft. Interestingly, the 18-year-old suture line (from the initial repair of aneurysm) was still clearly visible in the aorta because of the lack of connective tissue overgrowth. A new prosthesis was anastomosed between the aorta just distal to the renal artery and the stent graft, which remained inside the original implanted Dacron graft (Fig. 3). Finally, the patient 


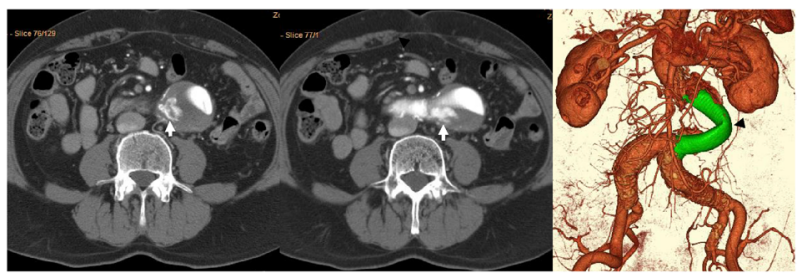

Fig. 1 Axial computed tomography (CT) scan before endovascular aneurysm repair reveals leakage (arrows) from the distal anastomosis of the Dacron graft, and a resulting pseudoaneurysm. Volume-rendered CT images clearly show a kink in the prosthesis (arrowhead). The site of the leak is unclear because of the patchy leakage pattern and the image reconstruction method.
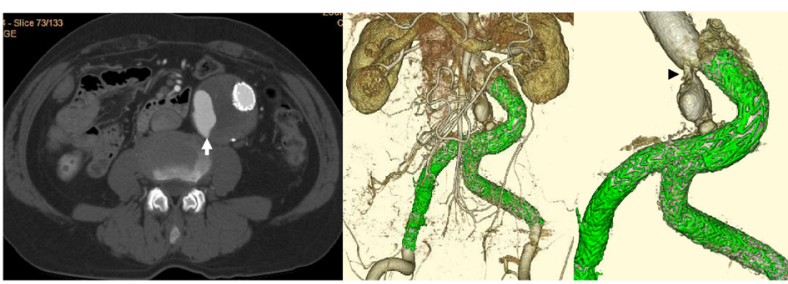

Fig. 2 Axial computed tomography (CT) scan 1 year after endovascular aneurysm repair reveals de-novo leakage from the proximal anastomosis of the Dacron graft and an increase in the aneurysm sac diameter (arrow). Volumerendered CT image clearly displays new leakage from the proximal anastomosis (arrowhead). It also shows that the kink in the prosthesis is smaller than before EVAR, possibly due to continuous exertion of radial force by the endograft and its tendency to return to a less deformed shape.

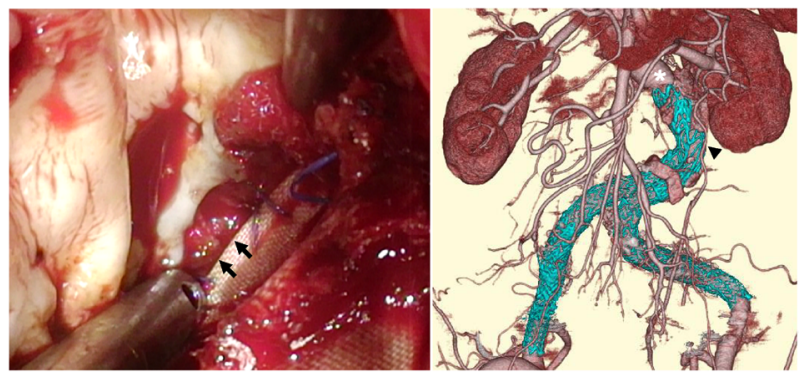

Fig. 3 Intraoperative photograph shows partial dehiscence of the suture line at the posterior region of the anastomosis of the Dacron graft (arrows), corresponding with the computed tomography (CT) scan in Fig. 3. There is no growth of connective tissue over the suture line. One year after repeat open surgery, a volume-rendered CT image shows that there is no arterial leakage or further reduction of the kink in the prosthesis because of its proximal extension (asterisk) and the rebound force of the stent graft (arrowhead).

recovered without complications, and postoperative CT monitoring for 3 years has confirmed a gradual decrease in the size of the aneurysm sac.

\section{Discussion}

Use of endoprostheses in patients with connective tissue diseases remains controversial because of the potential risk of aortic dilatation. In this study, we performed an endovascular treatment in a patient with Marfan syndrome, who required repeated aortic repair procedures because of leakage from the anastomoses of the original Dacron graft. We were reluctant to perform open surgery because we expected dense adhesions due to the three previous laparotomies, including one for ruptured abdominal aortic aneurysm. He received an aortic valve replacement with a mechanical prosthesis. Although EVAR is less invasive than open surgery and the Dacron graft seemed to provide a stable proximal landing zone, a suture line dehiscence originated at the proximal anastomosis of the Dacron graft after this procedure. The possible pathogenesis of dehiscence was considered next on the basis of the clinical course and operative findings.

We used the original Excluder device for EVAR, which has greater longitudinal elasticity than the newer conformable Gore TAG thoracic endoprosthesis (W. L. Gore \& Associates). However, stent grafts are designed to resist deformation when subjected to extreme bending. A longitudinally stiff Excluder endograft was placed into the kinked vascular prosthesis of our patient, presumably leading to marked longitudinal deformation of the stent graft, possibly due to the exerted continuous stress on the suture line while trying to return to its original shape. Also, two stent grafts were overlapped at the proximal end of the Dacron graft to prevent a type 1a endoleak, which resulted in greater stress in this patient. In fact, the implanted stent graft bending decreased following open surgery, presumably because of the rebound force generated in the stent graft (Fig. 3). We suspect that the continuous radial force on the proximal anastomosis due to the tendency of the stent graft to return to its original shape resulted in partial suture line dehiscence within 1 year after EVAR. ${ }^{2)}$ Dehiscence of an 18-year-old suture line was not covered with tissue growth, possibly because of the underlying connective tissue disease in our patient.

There are other possible causes for de-novo anastomotic leakage between native aorta and Dacron graft. Complications such as intimal tears and retrograde aortic dissection have been reported after endograft deployment in patients with subacute or chronic aortic dissection, particularly when an endograft is employed for aortic arch repair. Causative factors are believed to include hook injury and use of endografts that are too wide for the native aorta in addition to coexisting weakness of the intimal flap. ${ }^{3,4)}$ Although our patient with Marfan syndrome possibly had abnormal connective tissue, the only finding at the surgery was partial dehiscence of the suture line with 
no evidence of aortic dissection or intimal tear. During the endovascular procedure, we inflated the balloon inside the Dacron prosthesis and the endograft did not reach the native aorta. Moreover, postoperative CT did not reveal new leakage, indicating that the leak occurred in the chronic phase.

It has been reported that Marfan syndrome is frequently associated with dilatation of the aortic root and with aneurysm formation at an anastomosis site from a previous root surgery. ${ }^{5,6)}$ However, infrarenal abdominal aortic aneurysm is relatively rare. ${ }^{7,8)}$ Therefore, pseudoaneurysm formation at an anastomosis in the abdominal aorta is uncommon, and it is a possible unique point of this case.

Several successful cases of endovascular repair of the thoracic aorta in patients with Marfan syndrome have been reported.9) The 2014 European Society of Cardiology guidelines on the diagnosis and treatment of aortic diseases state that surgery should be preferred over endovascular procedures for the thoracic aorta in these patients; however, the guidelines do not mention abdominal aorta. ${ }^{10)}$ It has been proposed that endovascular repair can be used as a bail-out procedure in these patients or in special circumstances where the stent graft landing site is within the original Dacron graft. ${ }^{9}$ ) However, our experience suggests that an endograft with longitudinal deformation may increase the risk of suture line dehiscence in patients with connective tissue disorders, even if it is landed within a prior Dacron graft. Selecting a device of the appropriate size that can accommodate aortic tortuosity by employing gentle maneuvers is mandatory if an endograft is used.

\section{Conclusion}

Endovascular repair is a useful and less invasive strategy for patients who require repeated aneurysm repair, and a Dacron graft may seem to provide a stable proximal landing zone. However, the strong tendency of some endograft devices to return to their original shape may apply stress that affects weakened regions of the native aorta, including anastomoses with prosthesis or sites of extreme kinking, particularly in patients with connective tissue diseases.

\section{Disclosure Statement}

All authors have no conflict of interest.

\section{Author Contributions}

\author{
Data collection: $\mathrm{HK}, \mathrm{NH}$ \\ Writing: HK \\ Final approval: all authors
}

\section{References}

1) Kasahara H, Shimizu H, Yozu R. Postoperative renal function after juxtarenal aortic aneurysm repair with simple cross-clamping. Ann Vasc Surg 2013; 27: 291-8.

2) Demanget N, Avril S, Badel P, et al. Computational comparison of the bending behavior of aortic stent-grafts. J Mech Behav Biomed Mater 2012; 5: 272-82.

3) Eggebrecht H, Thompson M, Rousseau H, et al.; European Registry on Endovascular Aortic Repair Complications. Retrograde ascending aortic dissection during or after thoracic aortic stent graft placement: insight from the European registry on endovascular aortic repair complications. Circulation 2009; 120 Suppl: S276-81.

4) Kpodonu J, Preventza O, Ramaiah VG, et al. Retrograde type A dissection after endovascular stenting of the descending thoracic aorta. Is the risk real? Eur J Cardiothorac Surg 2008; 33: 1014-8.

5) Taniguchi K, Nakano S, Matsuda H, et al. Long-term survival and complications after composite graft replacement for ascending aortic aneurysm associated with aortic regurgitation. Circulation 1991; 84 Suppl: III31-9.

6) Kitahara H, Yoshitake A, Hachiya T, et al. Hybrid thoracic endovascular aortic repair for intercostal patch aneurysm after thoracoabdominal aortic replacement. Ann Vasc Dis 2015; 8: 340-2.

7) Hagerty T, Geraghty P, Braverman AC. Abdominal aortic aneurysm in Marfan syndrome. Ann Vasc Surg 2017; 40: 294.e1-6.

8) Takayama T, Miyata T, Nagawa H. True abdominal aortic aneurysm in Marfan syndrome. J Vasc Surg 2009; 49: 11625.

9) Preventza O, Mohammed S, Cheong BY, et al. Endovascular therapy in patients with genetically triggered thoracic aortic disease: applications and short- and mid-term outcomes. Eur J Cardiothorac Surg 2014; 46: 248-53; discussion, 253.

10) Erbel R, Aboyans V, Boileau C, et al. 2014 ESC Guidelines on the diagnosis and treatment of aortic diseases: document covering acute and chronic aortic diseases of the thoracic and abdominal aorta of the adult. The Task Force for the Diagnosis and Treatment of Aortic Diseases of the European Society of Cardiology (ESC). Eur Heart J 2014; 35: 2873926. 